\title{
Maltol Promotes Mitophagy and Inhibits Oxidative Stress via the Nrf2/PINK1/Parkin Pathway after Spinal Cord Injury
}

\author{
Yuqin Mao, Jiqing Du $(\mathbb{D}$, Xianghang Chen, Abdullah Al Mamun $\mathbb{D}$, Lin Cao, Yanhong Yang, \\ Joana Mubwandarikwa, Muhammad Zaeem, Wanying Zhang, Yan Chen, Yusen Dai, \\ Jian Xiao $\mathbb{D}$, and Keyong Ye \\ Department of Orthopaedics, Affiliated Pingyang Hospital and School of Pharmaceutical Science, Wenzhou Medical University, \\ Wenzhou, Zhejiang 325000, China

\begin{abstract}
Correspondence should be addressed to Jiqing Du; jiqing.du@tum.de, Jian Xiao; xfxj2000@126.com,
\end{abstract} \\ and Keyong Ye; yky12600@126.com
}

Received 19 September 2021; Revised 5 January 2022; Accepted 6 January 2022; Published 1 February 2022

Academic Editor: Mateusz Maciejczyk

Copyright ( 2022 Yuqin Mao et al. This is an open access article distributed under the Creative Commons Attribution License, which permits unrestricted use, distribution, and reproduction in any medium, provided the original work is properly cited.

\begin{abstract}
Spinal cord injury (SCI), a fatal disease in the central nervous system, is characteristic of weak neuronal regeneration ability and complex pathological progress. Activation of oxidative stress (OS) and apoptosis-mediated cell death significantly contributes to the progression of SCI. Current evidence suggests that maltol exerts natural antioxidative properties via obstructing OS and apoptosis. However, the significant effect of maltol on SCI treatment has never been evaluated yet. In our current study, we explored maltol administration that could trigger the expression of Nrf2 and promote the retranslocation of Nrf2 from the cytosol to the nucleus, which can subsequently obstruct OS signal and apoptosis-mediated neuronal cell death after SCI. Furthermore, we found that maltol treatment enhances PINK1/Parkin-mediated mitophagy in PC12 cells, facilitating the recovery of mitochondrial functions. Our findings propose that maltol could be a promising therapeutic candidate for the treatment and management of SCI.
\end{abstract}

\section{Introduction}

As a destructive central nervous system injury, spinal cord injury (SCI) leads to a series of motor, sensory, and autonomic nervous dysfunctions. Surprisingly, recent data indicate that about 250,000 to 500,000 patients suffer from SCI worldwide every year, which poses a heavy economic and psychological burden $[1,2]$. The pathological process of SCI includes primary injury and secondary injury. The immediate injury is defined as the mechanical injury of the spinal cord. The secondary injuries including inflammation, ischemia, apoptosis, oxidative stress (OS), and mitochondrial dysfunctions further lead to severe tissue damage and inhibit the regeneration of the neuronal nerves in the injured area [3].

The SCI lesion can be accompanied by hypoxia-ischemia and inflammation, causing dysregulation of ion homeostasis, characterized by excessive ROS production, oxidative dam- age, and neuronal apoptosis. The secondary injurymediated neuronal apoptosis disrupts neural connection with sensory and motor dysfunctions. Increasing evidence suggests that suppression of the secondary injury-mediated neuronal apoptosis and obstructing the generation of OS deliver a promising therapeutic approach for the treatment and management of SCI $[2,4,5]$.

Mitophagy eliminates the damaged mitochondria and plays a pivotal role in maintaining mitochondrial homeostasis and neuronal cell survival [6]. PINK1/Parkin-mediated mitophagy is one of the essential mitophagy pathways [7]. PTEN-induced putative kinase 1 (PINK1) localizes on the outer membrane of the damaged mitochondria; subsequently, recruiting the Parkin protein from the cytoplasm to the mitochondria can activate Parkin protein for its downstream signaling. Parkin is an E3 ubiquitin ligase, which can contribute to the ubiquitination of mitochondrial membrane proteins and eliminate the damaged 

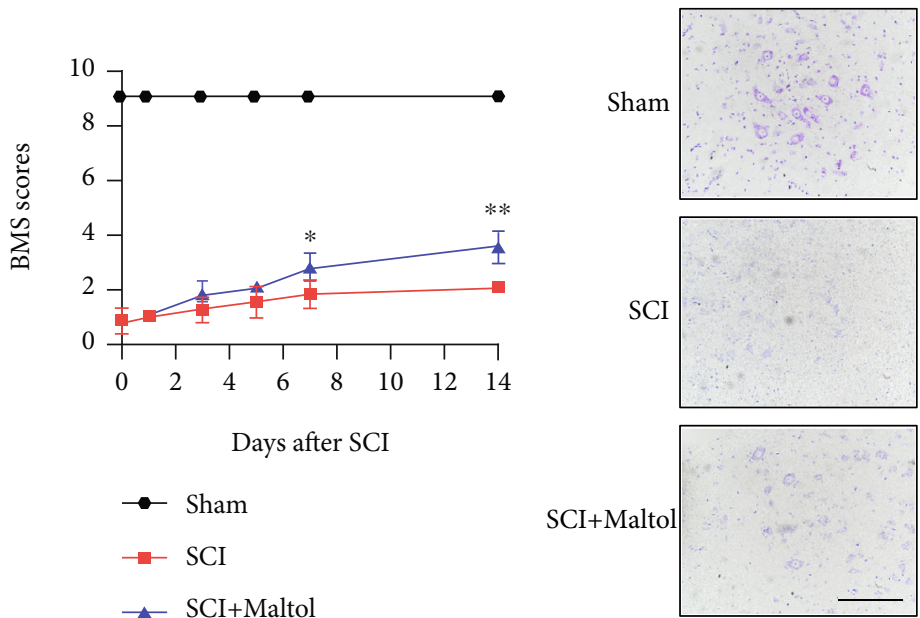

(a)

(b)
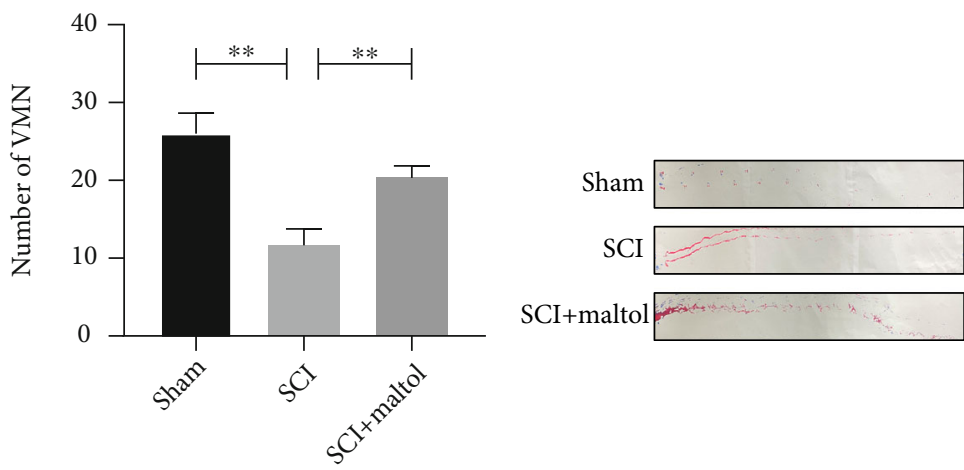

(c)

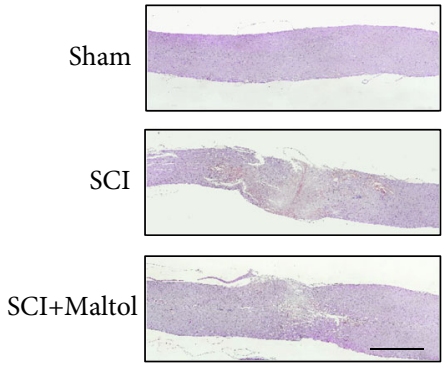

(d)

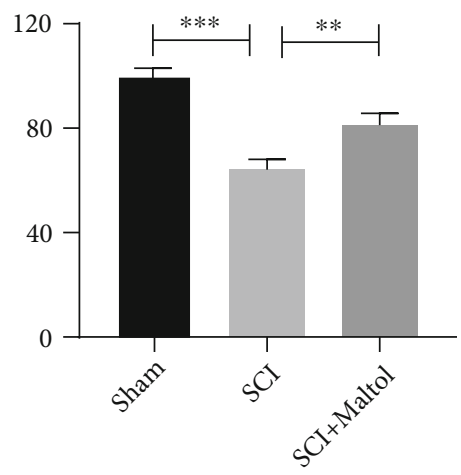

(e)

(f)

Figure 1: Maltol reduced spinal cord tissue damage and improved locomotor function after SCI. (a) Statistical analysis of BMS in each group from day 1 to day14 after SCI, $n=4$ per group. (b) Nissl staining sections of the spinal cord at 7 days after SCI. Scale bar: $100 \mu \mathrm{m}$ (20x). (c) Counting analysis of ventral motor neurons (VMN), $n=3$ per group. (d) Representative images of footprints in mice in each group at 14 days after SCI. (e) H\&E staining sections of the spinal cord at 7 days after SCI. Scale bar: $500 \mu \mathrm{m}$. (f) Quantitative analysis of preserved spinal cord tissue percentage, $n=3$ per group. Values were given as means $\pm \mathrm{SD} .{ }^{*} P<0.05,{ }^{* *} P<0.01,{ }^{* * *} P<0.001$.

mitochondrial substances by activating mitophagy [7, 8]. Mitochondrial functions can be improved by scavenging ROS and enhancing mitophagy [9]. Mounting evidence demonstrates that the promotion of mitophagy mitigates neuronal apoptosis and further restores neuronal functions after SCI $[6,10]$.

Nrf2 pathway activation can suppress OS-induced apoptosis and regulate mitochondrial functions via facilitating mitophagy [11, 12]. Nuclear erythroid 2-related factor 2 (Nrf2) is a crucial regulator against the activation of OS [13]. Under normal circumstances, the activity of $\mathrm{Nrf} 2$ is negatively regulated by the formation of Kelch-like ECHassociated protein 1 (Keap1) [14]. Nrf2 is released from Keap1, and the Nrf2 complex further translocates to the nucleus from the cytosol, activating the antioxidant response element (referred to as ARE hereafter). Increasing evidence 


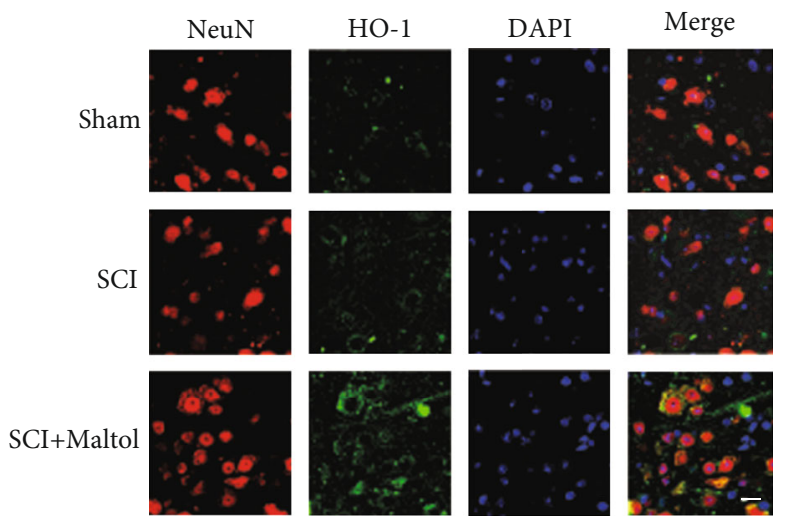

(a)

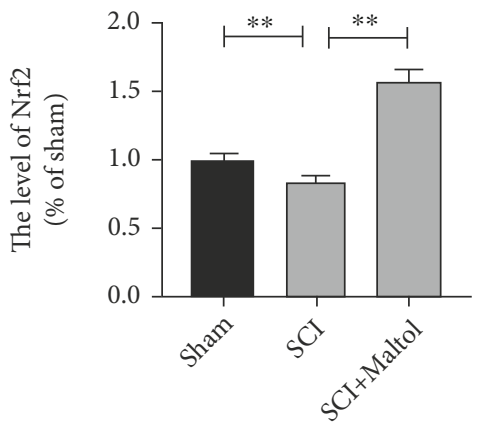

(c)

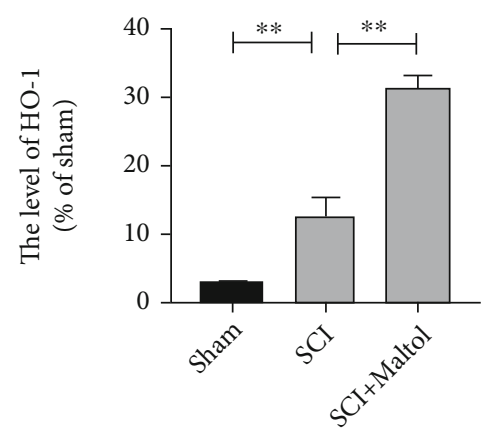

(d)

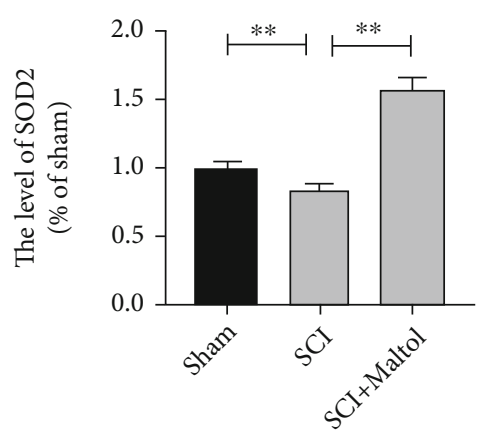

(e)

FIGURE 2: Maltol inhibited oxidative stress caused by SCI. (a) Coimmunofluorescence images show NeuN (red) and HO-1 (green) after SCI. Scale bar $=20 \mu \mathrm{m}$. (b-e) Western blots analysis of Nrf2, HO-1, and SOD2. $n=3$ per group. Values were indicated as means \pm SD. ${ }^{*} P<0.05$, ${ }^{* *} P<0.01$.

reports Nrf2 pathway activation triggers multiple genes to express the antioxidant-associated proteins such as heme oxygenase-1 (HO-1), $\mathrm{NAD}(\mathrm{P}) \mathrm{H}$ : quinone oxidoreductase 1 (NQO1), and superoxide dismutase 2 (SOD2) $[15,16]$.

Accumulating research suggests that some chemical antioxidants such as walnut-derived peptide and MitoQ can enhance mitophagy by obstructing OS and apoptosis via facilitating the Nrf2/PINK1/Parkin signaling pathway [17, 18]. However, maltol (3-hydroxy-2-methyl-4-pyrone, $\mathrm{C}_{6} \mathrm{H}_{6} \mathrm{O}_{3}$ ) is a Maillard reaction product by the pyrolysis of starch or sucrose and is widely applied as a reliable food additive and a natural antioxidative agent [19]. Previous studies have shown that maltol intervention could effectively protect peripheral nerves and suppress diabetic-mediated OS [20].
In addition, recent studies have reported that supplementation of maltol can activate the Nrf2/HO-1 signal pathway in the brain by suppressing OS and apoptosis-mediated cell death and alleviating D-Ga-induced neurological impairment 1 [21]. However, the potential antioxidative effect of maltol in targeting mitophagy and SCI has been elusive.

We performed mouse models and cell lines to evaluate the potential therapeutic effects of maltol on SCI. In our research, we found that maltol treatment could facilitate mitophagy and suppress the activation of OS and neuronal apoptosis via triggering the Nrf2/PINK1/Parkin signaling pathway after SCI. Therefore, our findings suggested that maltol could be a promising therapeutic candidate for the treatment of and management of SCI in the near future. 


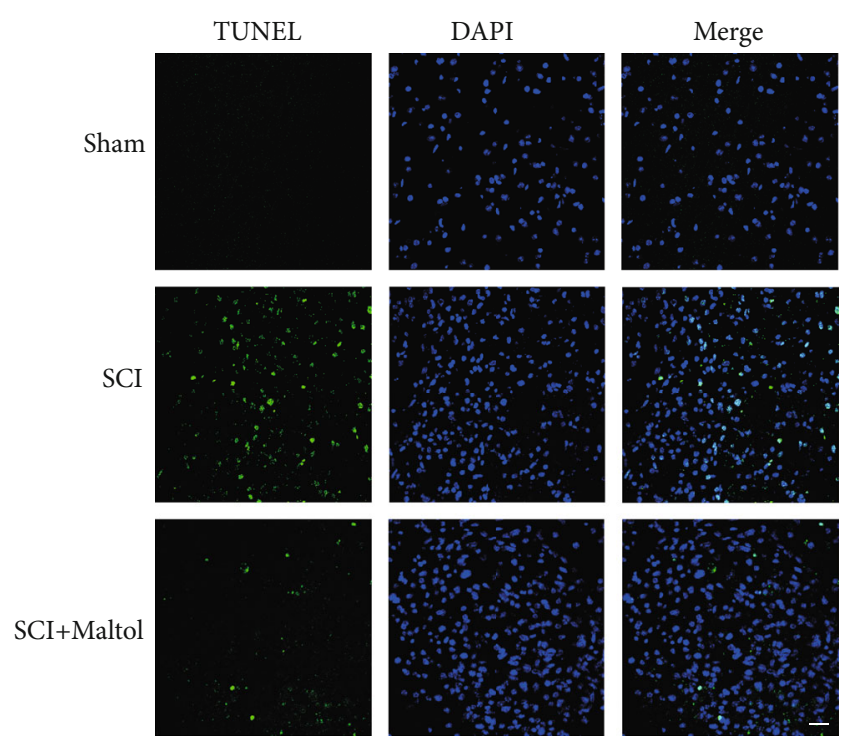

(a)

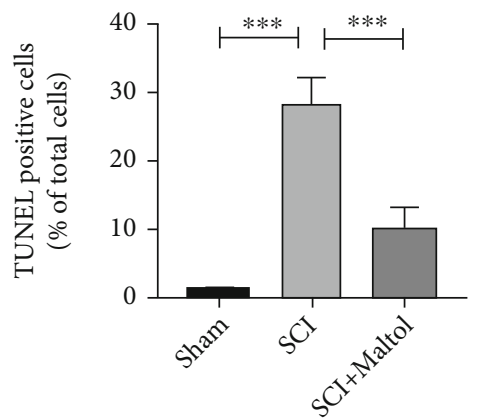

(b)
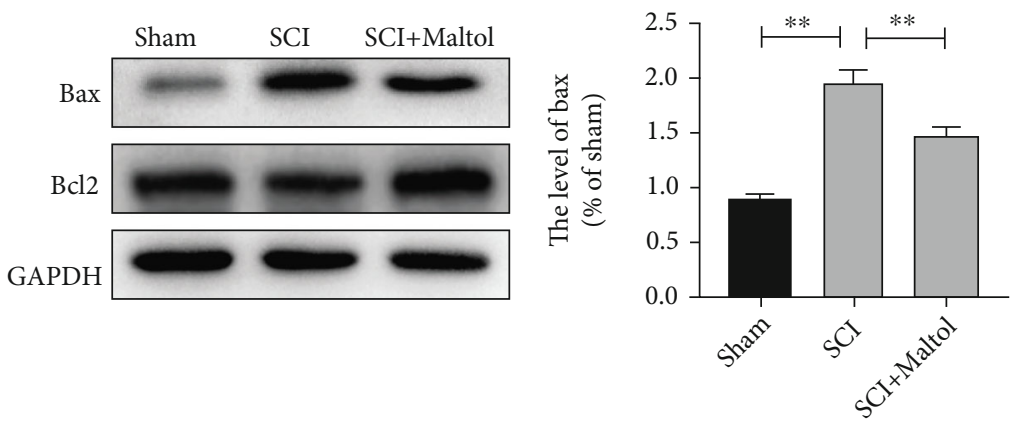

(c)

(d)

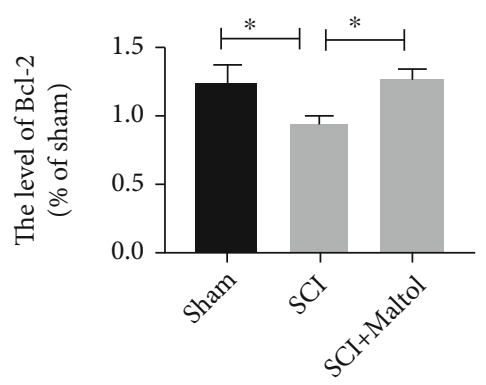

(e)

FIGURE 3: Maltol inhibited apoptosis caused by SCI. (a) TUNEL staining (green) after SCI. Scale bar $=50 \mu \mathrm{m}$. (b) Quantitative analysis of TUNEL-positive cells around the injury site, $n=3$ per group. (c-e) Western blots analysis of Bax and Bcl-2. $n=3$ per group. Values were given as means $\pm \mathrm{SD} .{ }^{*} P<0.05,{ }^{* *} P<0.01,{ }^{* * *} P<0.001$.

\section{Materials and Methods}

2.1. Animals and Ethics Statement. Sixty adult female 8week-old C57BL/6 mice (average weight: 20-25 g) were obtained from Wenzhou Medical University, Zhejiang province, China, for conducting our research. Under standard conditions including appropriate temperature $\left(23 \pm 2^{\circ} \mathrm{C}\right)$, humidity (50 $\pm 5 \%)$, and a 12 -hour light/12-hour dark cycle, all animals were placed in the OptiMICE Rotary Experimental Animal Cage System (Catalog No. C89100, Animal Care Systems, USA) and provided with adequate water and foods.
All the experimental procedures and protocols were strictly followed according to the guidance for the Animal Care and Use of Laboratory protocols of the National Institutes of Health and finally approved by the Animal Care and Use Committee of Wenzhou Medical University, Zhejiang Province, China.

2.2. Contusive SCI Model and Treatment. $40 \mathrm{mg} / \mathrm{kg}$ pentobarbital sodium $4 \%(w / v)$ was intraperitoneally administered in mice, and laminectomy was performed to remove the part of the vertebra covering the T9-T10 segment of 


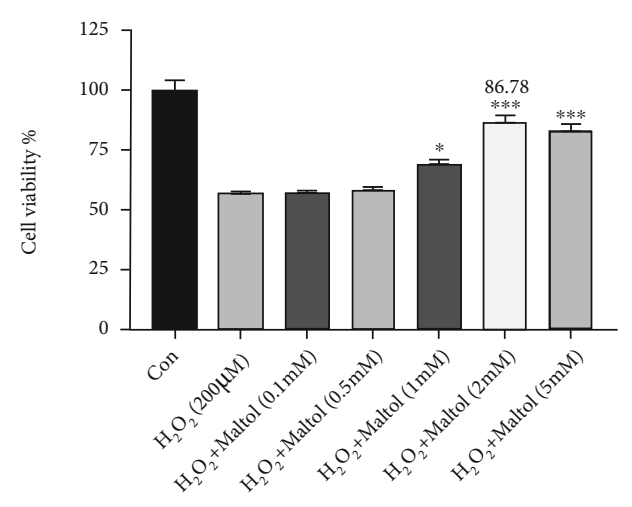

(a)

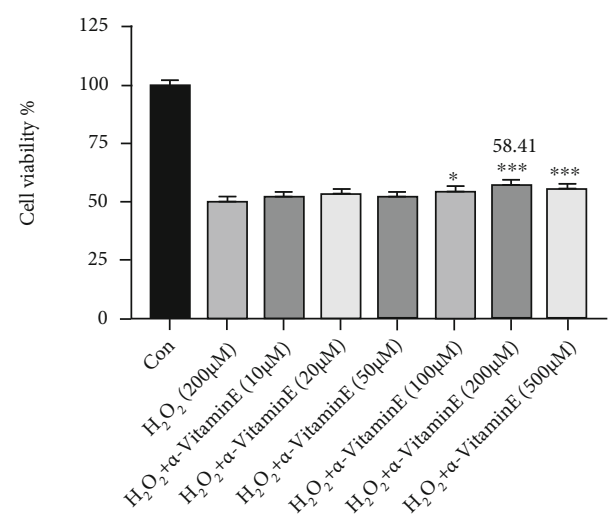

(c)

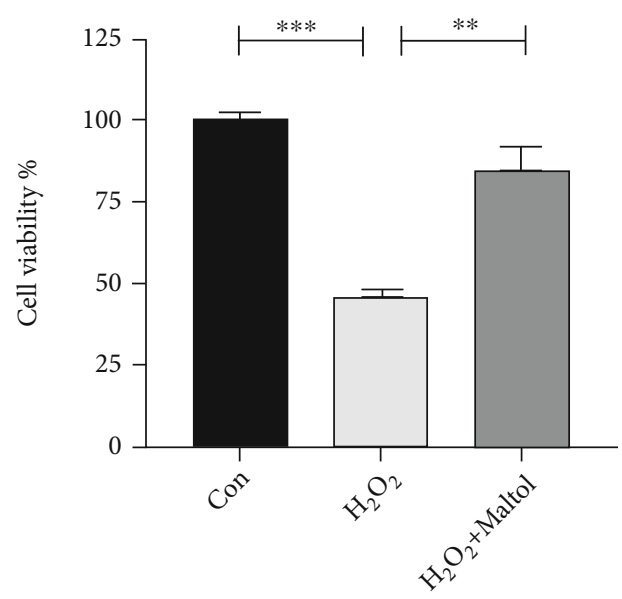

(e)

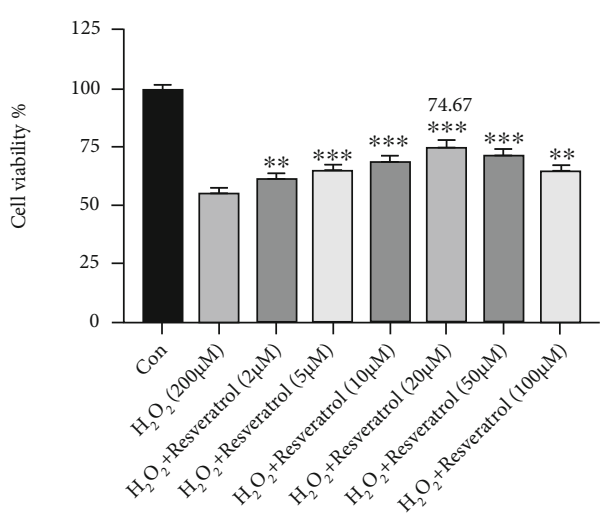

(b)

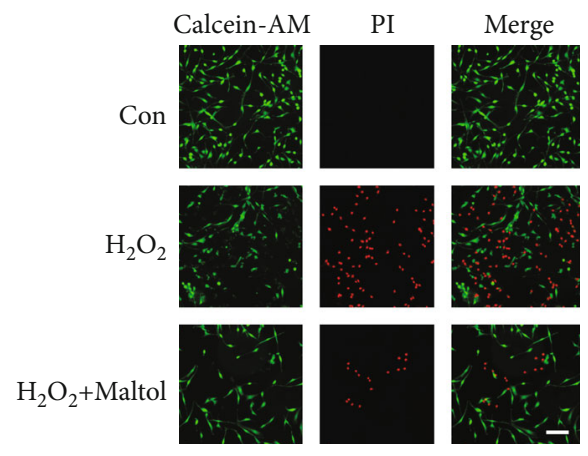

(d)

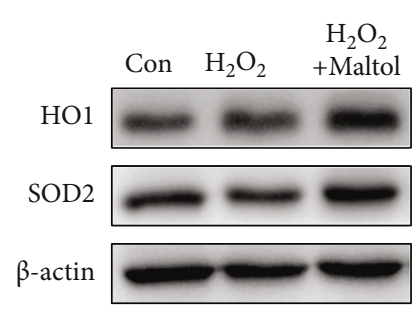

(f)

Figure 4: Continued. 


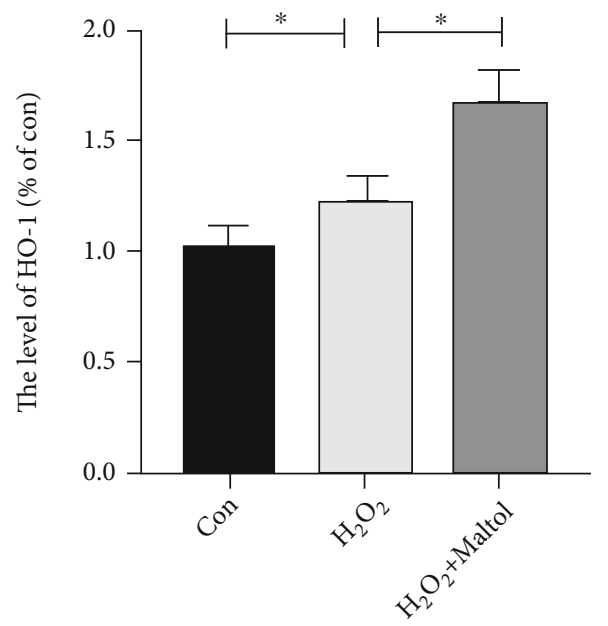

(g)

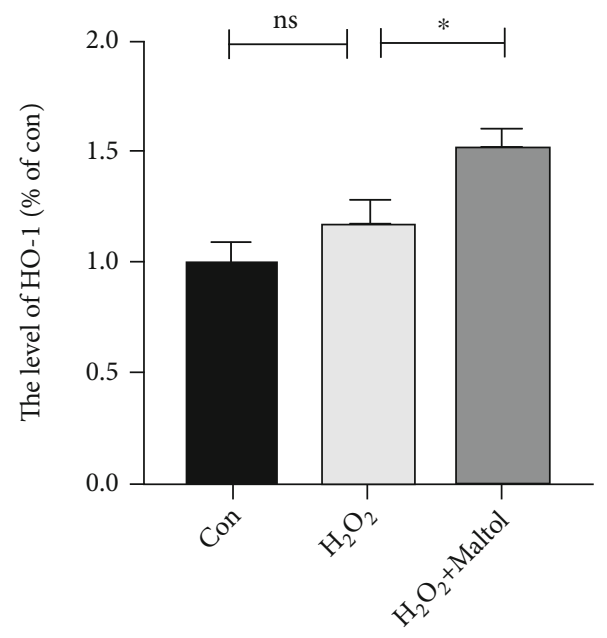

(h)

FIgure 4: Maltol attenuated $\mathrm{H}_{2} \mathrm{O}_{2}$-induced oxidative stress and apoptosis in PC12 cells. (a-c) Adopting CCK-8 to measure relative cell viability after being treated with maltol, resveratrol, and $\alpha$-vitamin $\mathrm{E}$ in different concentrations. $n=4$ per group. ${ }^{*} P<0.05,{ }^{* *} P<0.01$, ${ }^{* * *} P<0.001$ vs. $\mathrm{H}_{2} \mathrm{O}_{2}$ group. (d-e) Adopting the Calcein-AM/PI Double Stain Kit to distinguish live cells (green) from dead cells (red) and calculate cell viability. Scale bar $=100 \mu \mathrm{m}$. (f-h) Western blots analysis of HO-1 and SOD2 in vitro experiments. $n=3$ per group. Values were given as means $\pm \mathrm{SD} .{ }^{*} P<0.05,{ }^{* *} P<0.01,{ }^{* *} P<0.001$, and NS (not significant).

the spinal cord. Then, a $10 \mathrm{~g}$ weight steel rod was adopted to damage the exposed spinal cord from $20 \mathrm{~mm}$ height, resulting in a moderate contusive SCI model $[22,23]$. After the surgical operation, the bladders of the mice were manually emptied twice a day until the bladder's function recovered. All experimental mice were euthanized with excessive pentobarbital sodium on the $3 \mathrm{rd}, 7 \mathrm{th}$, and 14 th days after surgery. The animals were operated on to expose the spinal cord without injury for the sham group. For the SCI + maltol group, maltol (MedChemExpress, USA), $100 \mathrm{mg} / \mathrm{kg}$ dose (dissolved in normal saline), was administered to mice one time every day by gavage.

2.3. Cell Culture and Treatment. PC12 cells are widely used as an in vitro model to simulate neurons [24]. PC12 cells were purchased from the Cell Storage Center of Wuhan University, Wuhan, China. PC12 cells were carefully cultured and propagated in a suitable environment at $37^{\circ} \mathrm{C}$ with $5 \% \mathrm{CO}_{2}$ and $95 \%$ air. PC12 cells were cultured in RPMI1640 medium supplemented with 10\% fetal bovine serum (FBS, Invitrogen, Carlsbad, CA, USA), $100 \mathrm{U} / \mathrm{mL}$ penicillin, and $100 \mathrm{U} / \mathrm{mL}$ streptomycin. Control cells were cultured in RPMI1640 complete medium. For the $\mathrm{H}_{2} \mathrm{O}_{2}$ group, $\mathrm{H}_{2} \mathrm{O}_{2}$ (the $\mathrm{OS}$ inducer, $200 \mu \mathrm{M})$ was incubated to simulate OS for $2 \mathrm{~h}$. To assess the impact of maltol and Nrf2 signal, maltol $(2 \mathrm{mM})$ and the Nrf2 inhibitor ML385 (5 $\mu \mathrm{M}, \mathrm{HY}-100523$, MedChemExpress, USA) were applied for $12 \mathrm{~h}$ before $\mathrm{H}_{2} \mathrm{O}_{2}$ treatment.

2.4. Locomotion Recovery Assessment. A locomotion recovery assessment was performed at $0,1,3,7$, and 14 days (dpi) after SCI, mainly based on the Basso-Mouse-Scale (BMS) score. The BMS scoring ranged from 0 to 9, which primarily evaluates the function of the hind limbs of the experimental mice, consisting of ankle mobility, paw position, coordination, trunk stability, and tail position.
2.5. Tissue Preparation. Mice were euthanized at designated time points, and then ventricular perfusion was performed with normal saline solution. The $10 \mathrm{~mm}$-long spinal cord tissues were collected and immediately stored in a refrigerator at $-80^{\circ} \mathrm{C}$ for extracting proteins by western blotting. For histopathological and another staining, the ventricles of mice were perfused with normal saline and $4 \%$ paraformaldehyde (PFA). $10 \mathrm{~mm}$-long spinal cords were collected and immediately fixed in a $4 \%$ PFA for $48 \mathrm{~h}$. Then, the fixed spinal cords were dehydrated, embedded in paraffin, and cut into $5 \mu \mathrm{m}$ sections for subsequent staining.

2.6. Hematoxylin and Eosin (HßE) and Nissl Stainings. $5 \mu \mathrm{m}$ spinal cord tissue sections were deparaffinized, hydrated, and stained with Hematoxylin-Eosin and Nissl staining commercial kits (Solarbio, China), according to the manufacturer instructions. Finally, the tissue sections were evaluated under a light microscope (Nikon, Japan).

2.7. TUNEL Staining. The TUNEL Apoptosis Detection Kit (40307ES20, Yeasen Biochemical) was employed to measure the levels of apoptosis. According to the manufacturer's instructions, TUNEL staining was performed at $7 \mathrm{~d}$ after SCI. The section slides ( $5 \mu \mathrm{m}$ thick) were adequately deparaffinized and rehydrated. The slides were then carefully stained with a cell death detection kit (Roche, Basel, Switzerland) for $30 \mathrm{~min}$ at $37^{\circ} \mathrm{C}$. The 4,6-diamidino-2-phenylindole (DAPI) reagent was applied for the nuclei staining. Three independent and blinded observers chose twenty fields of each sample and captured images under a confocal microscope (Nikon, Japan).

2.8. Cell Viability Assay. Cell viability was performed by Cell Counting Kit-8 (C0043, Beyotime, China), according to the manufacturer's protocols. PC12 cells were inoculated in 


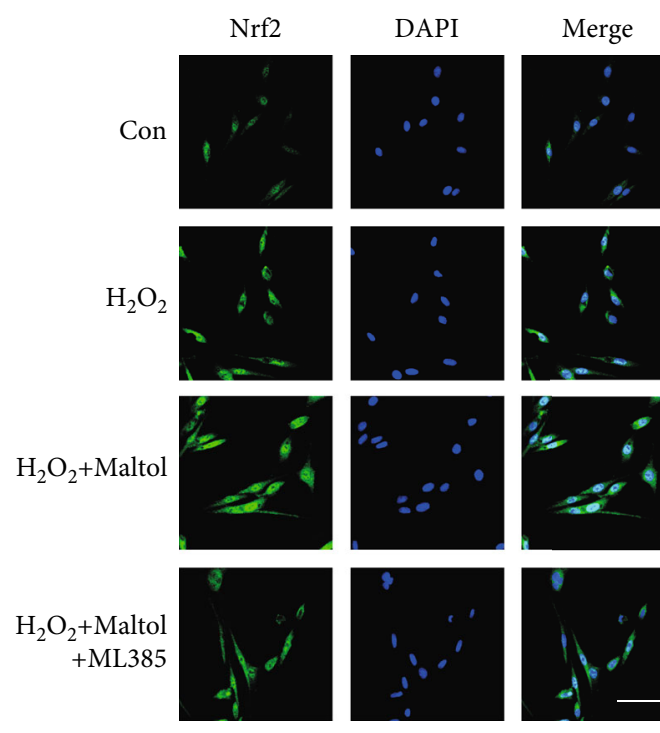

(a)

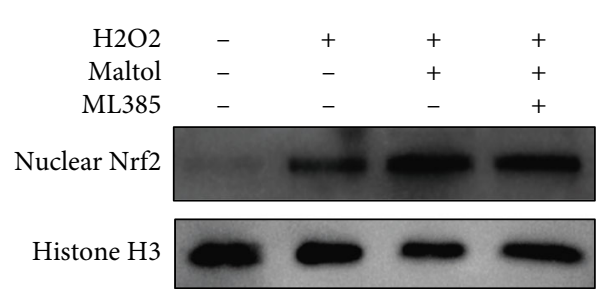

(b)

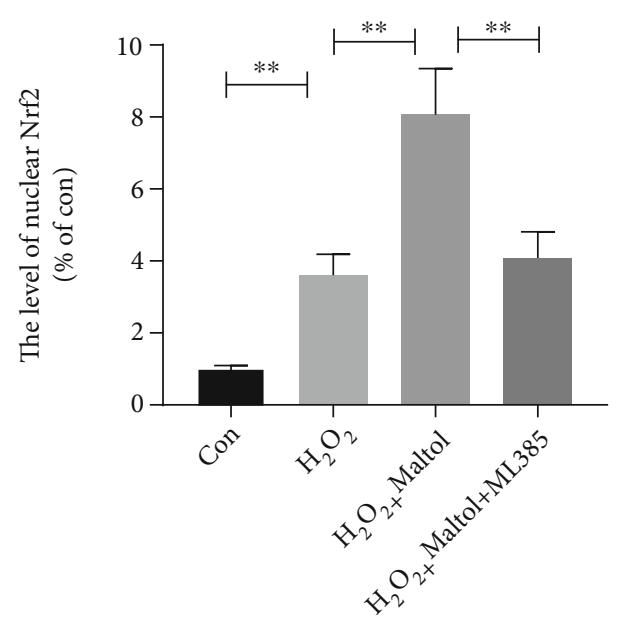

(c)

Figure 5: Maltol activated the Nrf2 signaling pathway in PC12 cells. (a) Immunofluorescence staining of Nrf2 (green) in PC12 cells. Scale bar $=50 \mu \mathrm{m}$. (b, c) Western blots analysis of Nrf2 in the nucleus. $n=3$ per group. Values were given as means \pm SD. ${ }^{* *} P<0.01$.

96-well plates $\left(8 \times 10^{3}\right.$ cells per well $)$ and cultured for $24 \mathrm{~h}$. Then, the cells were treated with drugs according to the experimental design. After that, $10 \mu \mathrm{L}$ of CCK- 8 solution was added with a $90 \mu \mathrm{L}$ basal medium to each well. After $1 \mathrm{~h}$, the absorbance at $450 \mathrm{~nm}$ of each sample was measured by a microplate reader.

The Calcein-AM/PI Double Stain Kit (40747ES76, Yeasen, China) is widely performed to detect live cells from dead cells. PC12 cells were incubated with $2 \mu \mathrm{M}$ Calcein-AM and $4.5 \mu \mathrm{M}$ PI for $30 \mathrm{~min}$ to label live cells and dead cells. Finally, the cells were observed by a confocal microscope (Nikon, Tokyo, Japan).

\subsection{Intracellular-ROS Generation and MitoSOX Staining.} Intracellular-ROS generation was measured by the ROS Assay Kit (S0033, Beyotime, China) and MitoSOX Red Mitochondrial Superoxide Indicator (40778ES50, YEASEN, China), according to the manufacturer's protocols. After being treated with drugs, PC12 cells were incubated with
$10 \mu \mathrm{M}$ DCFH-DA $(1: 1000)$ for $30 \mathrm{~min} / 5 \mu \mathrm{M}$ MitoSOX $(1: 1000)$ in the dark for $40 \mathrm{~min}$ at $37^{\circ} \mathrm{C}$ and washed three times with PBS. The nuclei were then labeled with the DAPI reagent and finally observed by a confocal microscope (Nikon, Tokyo, Japan).

2.10. Western Blot Analysis. Spinal cord tissues were collected 3 and 7 days after injury and immediately stored at $-80^{\circ} \mathrm{C}$ for western blot analysis. Spinal tissues and PC12 cells were lysed in ice-cold RIPA buffer solution with $1 \mathrm{mM}$ PMSF (phenyl methane sulfonyl fluoride, Beyotime). To measure the protein concentration of each sample, the BCA protein assay kit (Beyotime) was used according to the manufacturer's protocols. After that, the protein samples were separated via sodium dodecyl sulfate-polyacrylamide (SDS) gel electrophoresis and then carefully transferred to polyvinylidene difluoride membrane (PVDF) (Millipore, USA). The membrane proteins were sufficiently blocked with a $5 \%$ skim-milk solution at room temperature for $90 \mathrm{~min}$. Then, 


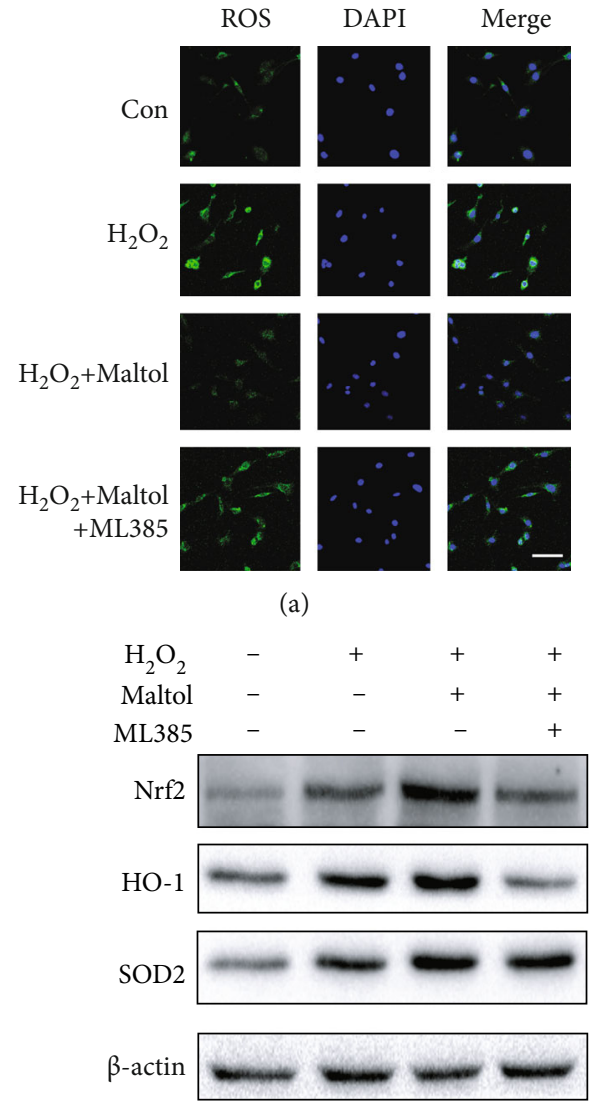

(c)

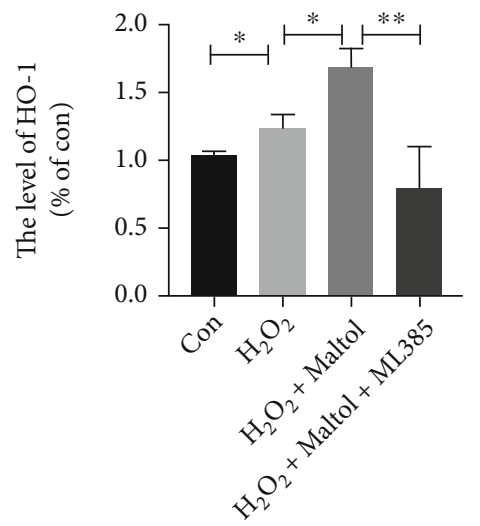

(e)

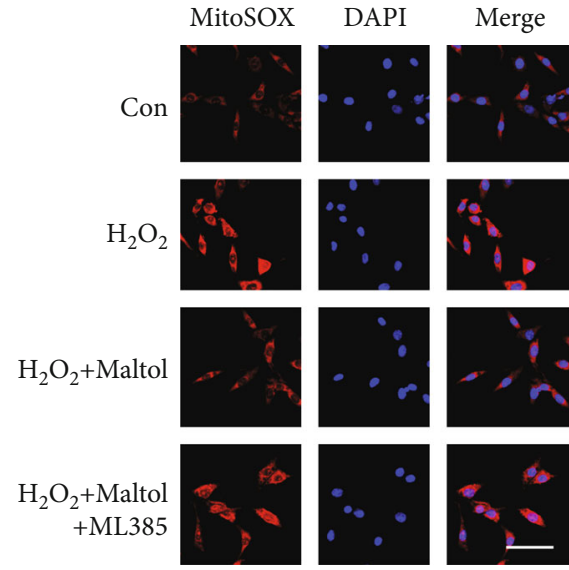

(b)

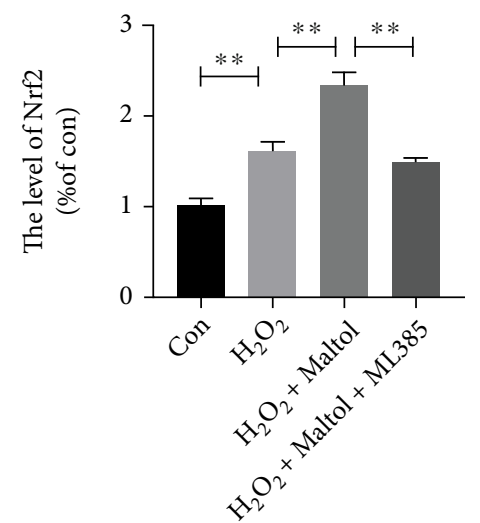

(d)

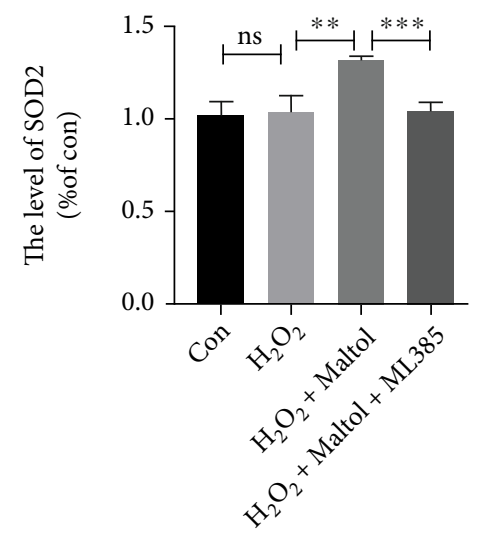

(f)

FIGURE 6: Maltol attenuated oxidative stress by activating the Nrf2/ARE pathway in PC12 cells. (a) Detection of ROS (green) production by the ROS Assay Kit in PC12 cells. Scale bar $=50 \mu \mathrm{m}$. (b) MitoSOX staining (red) in PC12 cells. Scale bar $=50 \mu \mathrm{m}$. (c-f) Western blots analysis of Nrf2, HO-1, and SOD2. $n=3$ per group. Values were given as means \pm SD. ${ }^{*} P<0.05,{ }^{* *} P<0.01,{ }^{* * *} P<0.001$, and NS (not significant).

the membranes were cut and incubated with specific primary antibodies against Nrf2 (1:1000, 16396-1-AP, ProteinTech, USA), HO-1 (1:1000, 10701-1-AP, ProteinTech, USA), SOD2 (1:2000, 24127-1-AP, ProteinTech, USA), $\beta$-actin ( $1: 1000,20536-1-A P$, ProteinTech, USA), GAPDH $(1: 1000$, 10494-1-AP, ProteinTech, USA), Bax (1:1000, 50599-2-Ig, ProteinTech, USA), Bcl-2 (1:1000, 26593-1-AP, ProteinTech, USA), Histone-H3 (1:1000, 17168-1-AP, ProteinTech, USA),
LC3 (1:1000, 14600-1-AP, ProteinTech, USA), PINK1 (1:1000, 23274-1-AP, ProteinTech, USA), Parkin (1:1000, 14060-1-AP, ProteinTech, USA), VDAC1 (1:1000, 380506, Zen-Bio, China), and TOM20 (1:1000, R25952, Zen-Bio, China) for overnight at $4^{\circ} \mathrm{C}$. Next, the membranes were incubated with horseradish peroxidase-conjugated secondary (HRP) antibodies (SA00001-1/SA00001-2, ProteinTech, USA) for $90 \mathrm{~min}$ at room temperature. The protein bands 


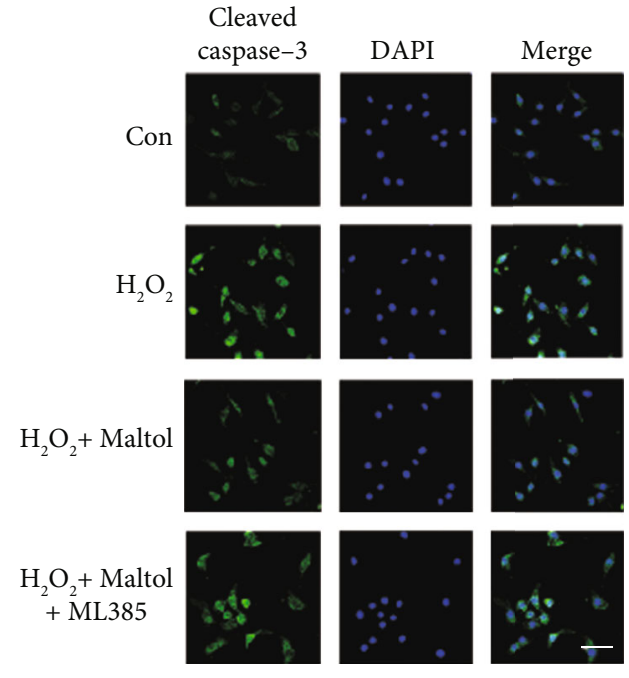

(a)

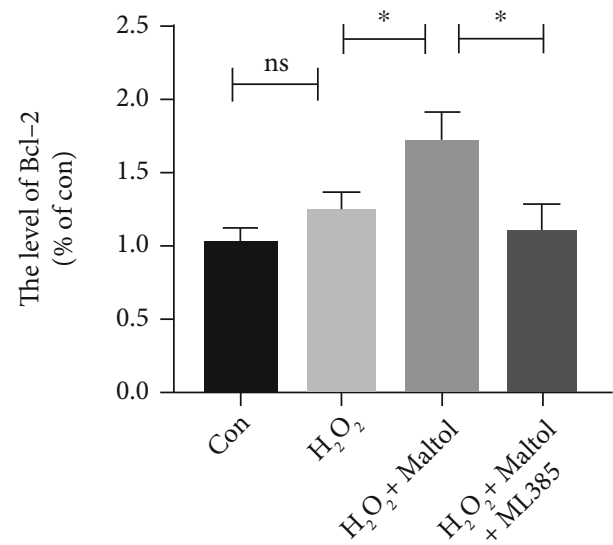

(c)

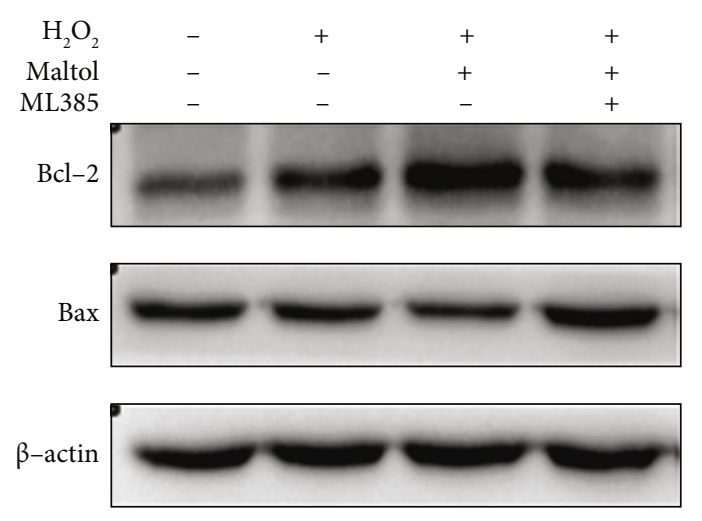

(b)

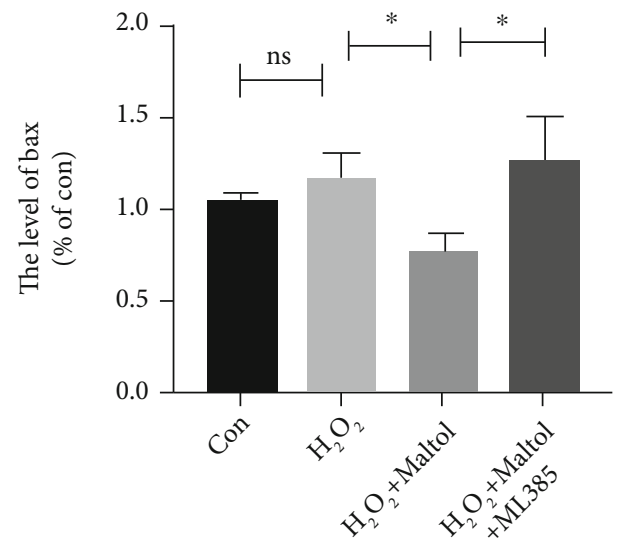

(d)

FIgURe 7: Maltol attenuated apoptosis by activating the Nrf2/ARE pathway in PC12 cells. (a) Immunofluorescence staining of cleaved caspase-3 (green) in PC12 cells. Scale bar $=50 \mu \mathrm{m}$. (b-d) Western blots analysis of Bax and Bcl-2. $n=3$ per group. Values were given as means \pm SD. ${ }^{*} P<0.05$ and NS (not significant).

were carefully evaluated by a ChemiDicTM XRS + Imaging System (Bio-Rad, Berkeley, California, USA). Finally, the bands were quantified using densitometric measurement with the Image Lab 3.0 software.

2.11. Immunofluorescence Staining. The spinal cord tissue sections were properly washed in PBS and carefully fixed with $4 \%$ paraformaldehyde solution. Then, the tissue slides were carefully permeated in $0.1 \%$ Triton X-100 solution for $3 \mathrm{~min}$. Then, the sample slides were adequately blocked with $5 \%$ bovine serum albumin (BSA) for $30 \mathrm{~min}$ at $37^{\circ} \mathrm{C}$. After adequate incubation, the slides were properly washed with PBS solution for 3 times. Then, the slides were incubated with specific primary antibodies including NeuN (1:1000, ab104224, Abcam, USA), HO-1 (1:300, 10701-1-AP, ProteinTech, USA), Nrf2 (1:300, 16396-1-AP, ProteinTech, USA), cleaved caspase-3 (1:1000, \#9661, CST, USA), and LC3B (1:1000, ab192890, Abcam, USA) for overnight at $4^{\circ} \mathrm{C}$. MitoTracker (M22426, Thermo Fisher, USA) was diluted with culture medium and then cocultured with living cells for 40 min. Later, Alexa Fluor 488/647-labeled secondary antibodies ( $1: 1000$, ab150077/ab150115, Abcam, USA) were applied to incubate sections and cells for $60 \mathrm{~min}$ at $37^{\circ} \mathrm{C}$. Then, the nuclei were stained with the DAPI reagent. The images were finally captured through a confocal laser microscope (Nikon, Tokyo, Japan).

2.12. Statistical Analysis. All data were expressed as the mean \pm SD. Experiments were repeated at least three times, and the tissues for each triplicate were from different mice. The statistical significances were determined by Student's $t$ -test and the one-way analysis of variance (ANOVA) test, followed by Tukey's multiple comparison test. A $P$ value < 0.05 was considered a significant value.

\section{Results}

3.1. Maltol Reduced Spinal Cord Tissue Damage and Improved Locomotor Function after SCI. To determine whether maltol administration alleviates functional recovery 


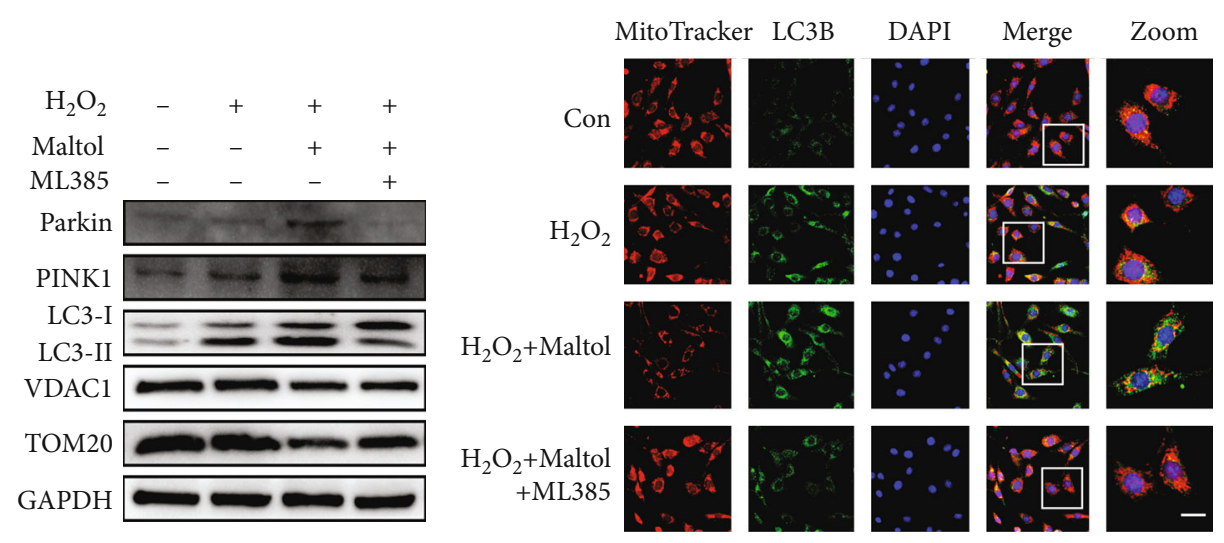

(a)

(b)

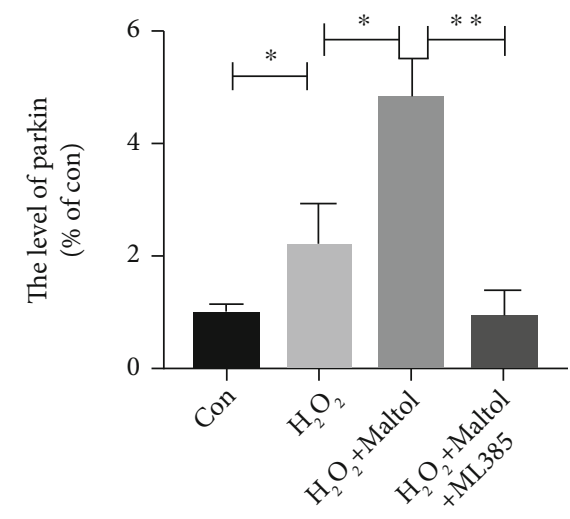

(c)

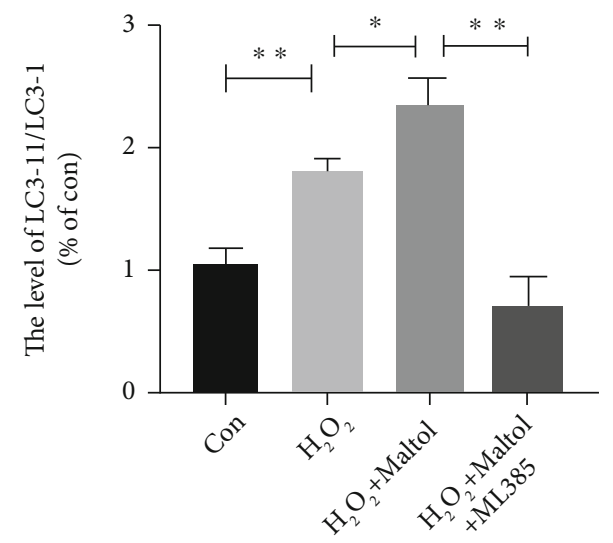

(e)

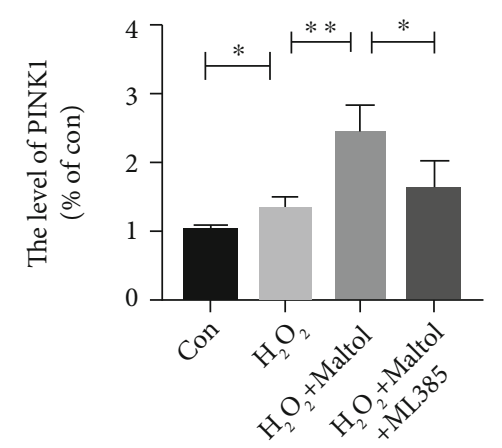

(d)

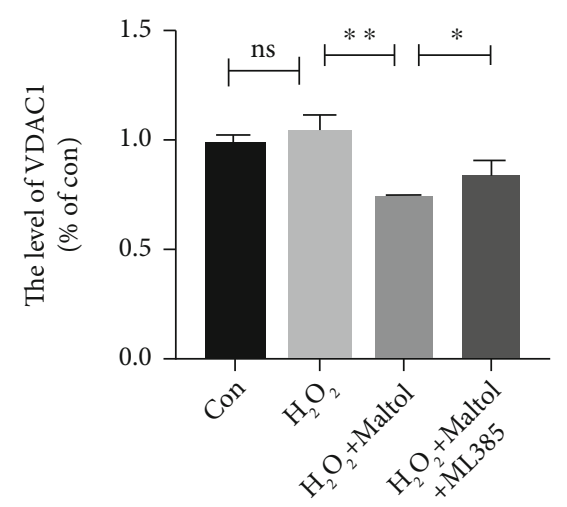

(f)

FIGURE 8: Continued. 


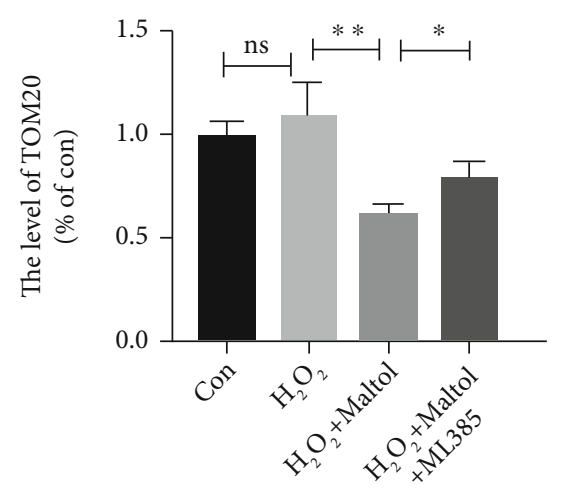

(g)

FIGURE 8: Maltol increased the level of mitophagy by activating the Nrf2/PINK1 signaling pathway in PC12 cells. (a, c-g) Western blots analysis of PINK1, Parkin, LC3, VDAC1, and TOM20. $n=3$ per group. (b) Coimmunofluorescence images show MitoTracker (red) and LC3B (green). Scale bar $=10 \mu \mathrm{m}$. Values were given as means \pm SD. ${ }^{*} P<0.05,{ }^{* *} P<0.01$, and NS (not significant).

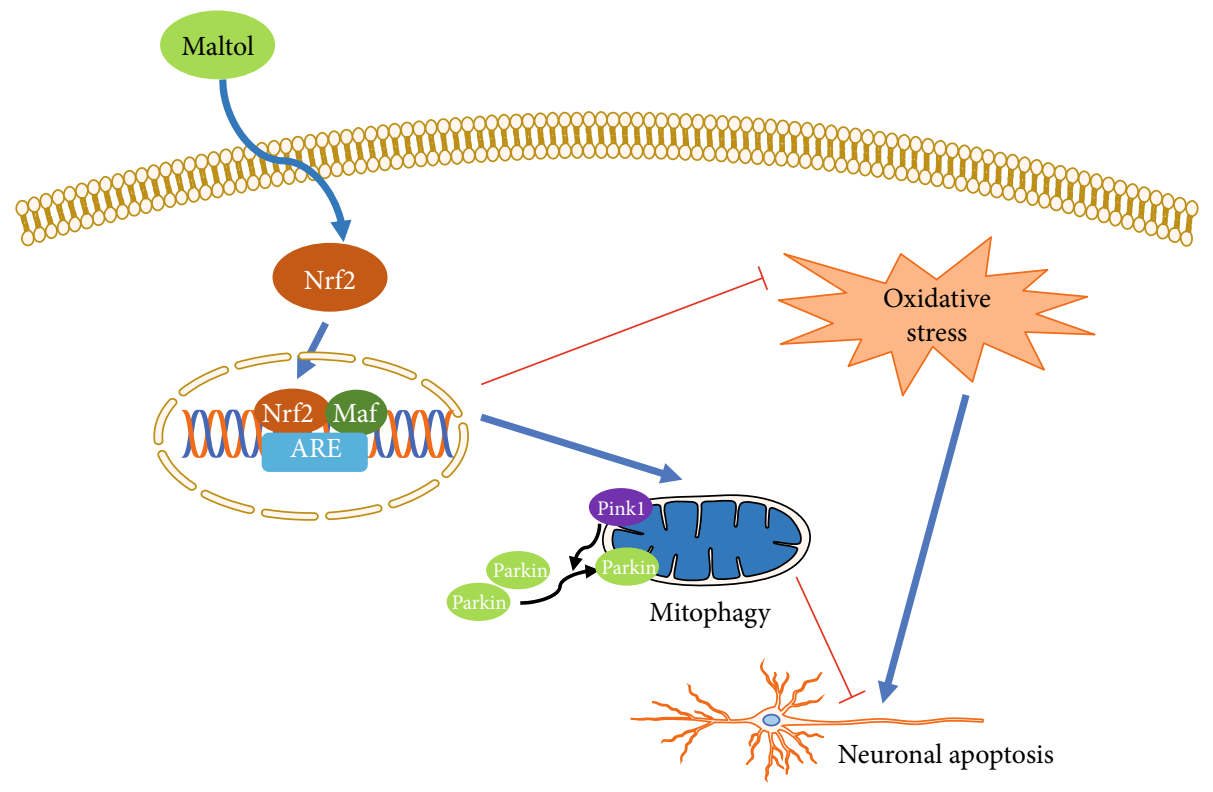

FIGURE 9: The potential molecular mechanism of maltol on SCI. Maltol can activate the expression of Nrf2 and facilitate Nrf2's translocation from the cytosol to the nuclear. Then it promotes mitophagy via enhancing the Nrf2/PINK1/Parkin pathway, ultimately suppressing the neuronal apoptosis in SCI.

after SCI, mice were administered with maltol $(100 \mathrm{mg} / \mathrm{kg} /$ day) by gavage after surgery. We conducted a 2-week BMS score to assess locomotor function after SCI. The results of the BMS analysis (Figure 1(a)) showed a significant reduction in score after SCI and gradually recovered after a few weeks. In the early stage of SCI (on the 1st and 3rd day), a significant difference between the SCI group and the SCI + maltol group was not noticeably observed, but in the late stage (on the 7 th and 14th day), the BMS scores of the maltol-treated SCI model group were remarkably higher than that of the SCI group, indicating that maltol administration could improve locomotor function after SCI. In addition, the representative patterns of the footprints also confirmed this situation (Figure 1(d)).
In addition, $\mathrm{H} \& \mathrm{E}$ and Nissl stainings were applied to observe the destruction of the spinal cord tissue and neuronal loss after SCI. Consistent with motor assessment, Nissl staining and neuronal counting assay indicated that maltol treatment significantly reduced the loss of ventral motor neurons in the SCI + maltol group compared with the SCI group (Figures 1(b) and 1(c)). Moreover, H\&E staining showed that maltol administration remarkably reduced peripheral blood and inflammatory cell infiltration, resulting in almost 2-fold reductions in the lesion area after SCI (Figures 1(e) and 1(f)).

3.2. Maltol Inhibited OS and Apoptosis Induced by SCI. Next, we measured the antioxidant and antiapoptotic capacity of maltol administration in SCI. Immunofluorescence findings 
showed that maltol administration could promote the expression of HO-1 in the neurons, suggesting that maltol improves the antioxidant ability of the neurons (Figure 2(a)). Compared with the nonmaltol mice group, western blot analysis clearly showed that maltol administration could facilitate the expressions of Nrf2 (Figures 2(b) and $2(\mathrm{c}))$ and the downstream antioxidant-related proteins such as HO-1 and SOD2 (Figures 2(b), 2(d), and 2(e)). TUNEL staining indicated that the level of apoptosis was significantly increased in the SCI mice model. In contrast, maltol administration could remarkably decrease apoptotic levels in mice with SCI (Figures 3(a) and 3(b)). Moreover, western blot analysis exhibited that maltol supplementation could downregulate the expression levels of proapoptotic protein Bax and upregulate the expression levels of antiapoptotic protein Bcl-2 in the injured mice model (Figures 3(c)-3(e)). Thus, these above findings suggested that maltol could restrict the activation of OS- and SCIinduced neuronal apoptosis.

3.3. Maltol Attenuated $\mathrm{H}_{2} \mathrm{O}_{2}$-Induced $\mathrm{OS}$ and Apoptosis in PC12 Cells. We then investigated whether maltol could alleviate $O S$ and apoptosis-mediated neuronal cell death in vitro experiment. PC12 cells are generally employed to simulate neurons in the central nervous system [24]. $\mathrm{H}_{2} \mathrm{O}_{2}$ is widely used to induce OS in cells. Our investigation found that the cell viability treated with maltol was positively correlated with the dose of maltol within a specific concentration range (0.1-2 mM). Cell viability assay revealed that maltol treatment at a dose-dependent manner could enhance cell viability rate in PC12 cells (Figure 4(a)).

Interestingly, compared to other natural antioxidants, resveratrol and $\alpha$-vitamin E, maltol treatment could significantly suppress OS-induced apoptosis. Primarily, compared with $\alpha$-vitamin $\mathrm{E}$, the cell viability with maltol treatment increased about 30\% (Figures 4(a)-4(c)). The Calcein-AM/ PI Double Stain results also confirmed that maltol treatment could reduce the apoptosis of PC12 cells (Figures 4(d) and $4(\mathrm{e})$ ). Our findings demonstrated that maltol could significantly inhibit $\mathrm{H}_{2} \mathrm{O}_{2}$-induced apoptosis. We further speculated that maltol upregulated the expression level of antioxidant proteins. Our western blot analysis revealed that maltol could promote the expressions of antioxidant-related proteins such as HO-1 and SOD2 (Figures 4(f)-4(h)).

3.4. Maltol Attenuated OS and Apoptosis by Activating the Nrf2/ARE Signaling Pathway in PC12 Cells. We further explored whether maltol treatment could promote the Nrf2 signaling pathway and obstruct the activation of OS and apoptosis-mediated cell death in PC12 cells. As shown by the immunofluorescence staining, maltol treatment increased the fluorescence intensity of Nrf2 and promoted the retranslocation of $\mathrm{Nrf} 2$ from the cytosol to the nucleus (Figure 5(a)). The application of ML385, a specific inhibitor of Nrf2, could reverse this potential effect. Meanwhile, the western blot assay also showed that maltol administration could facilitate the expression of $\mathrm{Nrf} 2$ in the nucleus (Figures 5(b) and 5(c)). Therefore, our findings suggested that maltol might significantly trigger the Nrf2 signaling pathway.

Next, we illustrated the connections between intracellular OS and the Nrf2 signaling pathway. We employed that $2^{\prime}, 7^{\prime}$-dichlorodihydrofluorescein diacetate (DCFH-DA) can be oxidized into fluorescent DCF by ROS, to assess ROS formation (Figure 6(a)). Our results showed that maltol treatment could dramatically decrease ROS formation in $\mathrm{H}_{2} \mathrm{O}_{2}$-stimulated PC12 cells. Significantly, ML385 reversed the inhibitory effect of maltol on ROS formation. Furthermore, we applied MitoSOX to detect mitochondrial superoxide and maltol could significantly restrict mitochondrial OS generation, indicating maltol delivers a protective effect on mitochondrial damage (Figure 6(b)). The western blot analysis exhibited that maltol could promote Nrf2, HO-1, and SOD2 in PC12 cells, while the application of ML385 decreased the expression of these antioxidant-related proteins (Figures 6(c)-6(f)). Therefore, maltol intervention could reduce ROS production and improve antioxidant capacity by enhancing the Nrf2 signaling pathway, which is consistent with the previous research [14].

We aimed to determine whether maltol could suppress apoptosis-mediated neuronal cell death via promoting the Nrf2 signaling pathway in PC12 cells. Cleaved caspase- 3 is widely applied to evaluate the level of apoptosis. Our immunofluorescence findings displayed that maltol treatment could remarkably reduce the fluorescence intensity of cleaved caspase-3. Furthermore, the application of ML385 could reverse the antiapoptotic effect of Nrf2, which further supported that Nrf2 suppressed apoptosis via enhancing its antioxidative effects (Figure $7(\mathrm{a})$ ). To further evaluate the antiapoptosis effect of maltol, we performed the western blot assay to determine the expression levels of the antiapoptotic protein $\mathrm{Bcl}-2$ and the proapoptotic protein Bax. Consistently, maltol treatment could downregulate the expression level of Bax and upregulate the expression level of Bcl-2 in PC12 cells, but ML385 reversely regulated the expression patterns of these two proteins (Figures $7(\mathrm{~b})-7(\mathrm{~d})$ ). In conclusion, these findings suggested that maltol could effectively suppress apoptosis via enhancing the Nrf2 signaling pathway.

3.5. Maltol Increased the Level of Mitophagy by Activating the Nrf2/PINK1/Parkin Signaling Pathway in PC12 Cells. We explored whether Nrf2 signaling pathway activation could facilitate mitophagy. Therefore, we first determined the expression levels of two well-known mitophagyassociated proteins such as PINK1 and Parkin. Western blot outcomes clearly demonstrated that the expression levels of these two proteins were dramatically upregulated in PC12 cells (Figures 8(a), 8(c), and 8(d)). Meanwhile, maltol treatment could reduce mitochondrial membrane proteins VDAC1 and TOM20 (Figures 8(a), 8(f), and 8(g)). Immunofluorescence findings showed reductions in the numbers of mitochondria (Figure 8(b)), indicating the activation of mitophagy. To confirm the event of mitophagy, we performed a confocal microscopic examination to ascertain the colocalization of LC3B (autophagosome) and MitoTracker (mitochondria). Surprisingly, immunofluorescence 
findings revealed that maltol treatment increased colocalization of LC3B with MitoTracker. Meanwhile, the application of ML385 in PC12 cells led to the mislocalization of LC3B and MitoTracker (Figure 8(b)). Furthermore, maltol treatment could increase the expression of LC3, and ML385 could inhibit the expression level of LC3 (Figures 8(a) and $8(\mathrm{e}))$.

\section{Discussion}

SCI is a severe central nervous system injury, increasing year by year with a tendency toward young people. The development of effective treatment has always been a significant challenge in neuroprotection and nerve regeneration after SCI $[2,25,26]$. The severe secondary damage caused by SCI including ischemia and hypoxia and inflammatory edema induces excessive ROS production, which eventually facilitates massive apoptosis-mediated neuronal cell death in the centre of the injury and surrounding areas, resulting in severe motor and sensory damage $[27,28]$. Therefore, it is an urgent need to develop effective drugs to treat and manage SCI.

Maltol, a product of the starch and sucrose pyrolysis process formed by the Maillard reaction, is widely used as a natural antioxidant [29, 30]. Numerous experiments have reported that maltol exerts significant antioxidant and antiapoptotic effects in treating and managing multiple diseases. In addition, a previous study revealed that maltol intervention promoted the recovery of peripheral nerves and alleviated diabetic peripheral neuropathy through suppressing OS and apoptosis [20]. Another study conducted by Liu and colleagues confirmed that maltol could facilitate the activities of glutathione (GSH) and superoxide dismutase (SOD) in the liver, promote HO-1 expression, and obstruct carbon tetrachloride-induced apoptosis [31]. However, no relevant studies have been reported on the protective effects of maltol and its specific mechanism to alleviate the progression of secondary SCI. Therefore, we aimed to investigate the protective mechanism of maltol in the treatment of SCI. To assess the significant effects of maltol in inhibiting the activation of OS, we compared maltol with other natural antioxidants. Compared with resveratrol and vitamin E, maltol could significantly suppress OS-driven apoptosis. Our present study further confirmed that maltol activated the Nrf2 signal and promoted PINK1/Parkin-mediated mitophagy to improve mitochondrial functions (Figure 9).

After SCI, spinal cord ischemia is accompanied by subsequent energy exhaustion and ATP deficiency, increasing intracellular calcium concentration and ultimately overproduction of ROS [32]. Excessive ROS formation increases the oxidative damage of proteins, DNA, and lipids molecules and promotes apoptosis-mediated cell death [33]. Our results suggested that gavage of maltol significantly promoted the expression of antioxidant-related proteins $\mathrm{HO}-1$ and SOD2 in the SCI mouse models. Furthermore, our immunofluorescence assay revealed that maltol supplementation could enhance the antioxidant capacity of neurons in the injured spinal cord tissue area. Meanwhile, we exposed PC12 cells to $\mathrm{H}_{2} \mathrm{O}_{2}$ to induce OS and treated them with maltol. Our results indicated that maltol could obstruct ROS and MitoSOX production in PC12 cells. Interestingly, our findings uncovered that inhibition of OS significantly attenuated neuronal apoptosis. In addition, our results demonstrated that oral administration of maltol significantly promoted the survival of neurons in mice and the survival of PC12 cells in vitro.

Nrf2 is a crucial transcription factor in the regulation of antioxidative stress. After binding to ARE in the nucleus, Nrf2 signals can initiate the expressions of a variety of antioxidant, anti-inflammatory, and antiapoptosis genes [34, 35]. Previous studies have shown that maltol activates the Nrf2 signaling pathway. Interestingly, Sha and coworkers demonstrated that maltol supplementation could reduce the levels of OS and restain apoptosis via promoting the Nrf2/HO-1 signaling axis in the brain tissue alleviating DGal-induced neurological impairment [21]. A pioneering research reports that maltol administration attenuates the aggravation of osteoarthritis via enhancing the Nrf2/HO-1 signaling pathway [36]. Our study is consistent with the above analysis that maltol can activate the Nrf2 signal pathway. Our findings revealed that maltol could promote the expression of Nrf2 in the spinal cord after SCI. In PC12 cells, maltol could dramatically boost the translocation of $\mathrm{Nrf} 2$ into the nucleus. Then the activated Nrf2 fosters the expression of HO-1 and SOD2, which was further confirmed by the application of the Nrf2 inhibitor.

Mitophagy is a process of the selective removal of damaged and senescent mitochondria and regulates mitochondrial quality and homeostasis [37]. Growing evidence indicates that PINK1/Parkin is a classical mitochondrial autophagic pathway $[8,38,39]$. During mitochondrial damage or ageing, the mitochondrial membrane potential decreases, and PINK1 accumulates in the outer membrane and promotes the activation and translocation of Parkin, which eventually leads to the degradation of mitochondrial proteins such as TOM20 and VDAC1 [40]. Emerging evidence revealed that $\mathrm{Nrf2}$, a transcription factor, regulates various cytoprotective genes and mitochondrial functions [41]. Nrf2 signaling pathway activation regulates membrane potential activity, antioxidant defense, biogenesis, and integrity in mitochondria [11, 42]. Recent literature reports that Nrf2 can remarkably trigger mitophagy through the Nrf2/ PINK1 signaling axis. Interestingly, Bertrand and colleagues found that the synthetic Nrf2 activator, 1,4-diphenyl-1,2,3triazole compound, promotes mitophagy and plays a contributory role in regulating mitochondrial homeostasis [43]. Current studies have found that some potential antioxidants such as walnut-derived peptide and MitoQ can facilitate PINK1/Parkin-mediated mitophagy and obstruct OS by activating the Nrf2 signaling pathway $[17,18]$. However, the potential targets of maltol on mitochondrial functions have not been well studied. Thus, we speculated that maltol could facilitate the Nrf2/PINK1/Parkin signaling pathway in SCI mouse models. Our results confirmed that maltol administration could trigger the Nrf2 pathway, which further promoted the expression of PINK1 and Parkin in PC12 cells. 
Interestingly, maltol treatment could reduce the expression levels of mitochondrial membrane proteins including TOM20 and VDAC1 in PC12 cells. Immunofluorescence findings clearly indicated that maltol could significantly facilitate mitochondrial function and autophagosome colocalization. In a word, our results strongly suggested that maltol administration could promote mitophagy in the SCI mice model. Our investigation also revealed that the application of Nrf2 inhibitor reversed the above effect.

Indeed, there are still some limitations in our current study. For instance, other aspects of mitochondrial functions including mitochondrial membrane potential and mitochondrial biogenesis have not been further investigated. More animal experiments might be needed to further prove the results of our cell experiments. In addition, the evaluation of the long-term effects of maltol intervention on SCI is also unknown in our current investigation. Further studies are highly warranted to explore the long-term pharmacological effects of maltol intervention in SCI. Mechanistically, our research found that maltol intervention could significantly promote mitophagy and obstruct OS signals and apoptosis-mediated neuronal cell death through enhancing the Nrf2/PINK1/Parkin pathway after SCI, suggesting that maltol could be a promising therapeutic agent for the treatment and management of SCI.

\section{Data Availability}

The original data supporting the conclusions of this article can be available from the corresponding authors upon request.

\section{Conflicts of Interest}

The authors declare no conflict of interest.

\section{Authors' Contributions}

Yuqin Mao, Jiqing Du, and Xianghang Chen contributed equally to this work.

\section{Acknowledgments}

This work was partially supported by a research grant from the National Natural Science Foundation of China (81972150, 82172428) and Zhejiang Provincial Natural Science Foundation (LR18H150001, LQ18H090008).

\section{References}

[1] M. Khorasanizadeh, M. Yousefifard, M. Eskian et al., "Neurological recovery following traumatic spinal cord injury: a systematic review and meta-analysis," Journal of Neurosurgery Spine, vol. 30, no. 5, pp. 683-699, 2019.

[2] A. Anjum, M. D. Yazid, M. Fauzi Daud et al., "Spinal cord injury: pathophysiology, multimolecular interactions, and underlying recovery mechanisms," International Journal of Molecular Sciences, vol. 21, no. 20, p. 7533, 2020.
[3] L. Isaac and L. Pejic, "Secondary mechanisms of spinal cord injury,” Surgical Neurology, vol. 43, no. 5, pp. 484-485, 1995.

[4] H. Zhang, D. Li, Y. Zhang et al., "Knockdown of lncRNA BDNF-AS suppresses neuronal cell apoptosis via downregulating miR-130b-5p target gene PRDM5 in acute spinal cord injury," RNA Biology, vol. 15, no. 8, pp. 1071-1080, 2018.

[5] Y. Rong, W. Liu, J. Wang et al., "Neural stem cell-derived small extracellular vesicles attenuate apoptosis and neuroinflammation after traumatic spinal cord injury by activating autophagy," Cell Death \& Disease, vol. 10, no. 5, p. 340, 2019.

[6] Q. Li, S. Gao, Z. Kang et al., "Rapamycin enhances mitophagy and attenuates apoptosis after spinal ischemia-reperfusion injury," Frontiers in Neuroscience, vol. 12, p. 865, 2018.

[7] D. Ivankovic, K. Y. Chau, A. H. Schapira, and M. E. Gegg, "Mitochondrial and lysosomal biogenesis are activated following PINK1/parkin-mediated mitophagy," Journal of Neurochemistry, vol. 136, no. 2, pp. 388-402, 2016.

[8] A. Eiyama and K. Okamoto, "PINK1/Parkin-mediated mitophagy in mammalian cells," Current Opinion in Cell Biology, vol. 33, pp. 95-101, 2015.

[9] B. Wang, M. Huang, D. Shang, X. Yan, B. Zhao, and X. Zhang, "Mitochondrial behavior in axon degeneration and regeneration," Frontiers in Aging Neuroscience, vol. 13, article 650038, 2021.

[10] C. Gu, L. Li, Y. Huang et al., "Salidroside ameliorates mitochondria-dependent neuronal apoptosis after spinal cord ischemia-reperfusion injury partially through inhibiting oxidative stress and promoting mitophagy," Oxidative Medicine and Cellular Longevity, vol. 2020, Article ID 3549704, 2020.

[11] A. T. Dinkova-Kostova and A. Y. Abramov, "The emerging role of Nrf2 in mitochondrial function," Free Radical Biology \& Medicine, vol. 88, no. Part B, pp. 179-188, 2015.

[12] T. Y. Zhang, P. Wu, E. Budbazar et al., "Mitophagy reduces oxidative stress via Keap1 (Kelch-like epichlorohydrinassociated protein 1)/Nrf2 (nuclear factor-E2-related factor 2)/PHB2 (prohibitin 2) pathway after subarachnoid hemorrhage in rats," Stroke, vol. 50, no. 4, pp. 978-988, 2019.

[13] L. Kang, Y. Tian, X. Guo, X. Chu, and Y. Xue, "Long noncoding RNA ANPODRT overexpression protects nucleus pulposus cells from oxidative stress and apoptosis by activating Keap1-Nrf2 signaling," Oxidative Medicine and Cellular Longevity, vol. 2021, 2021.

[14] I. Bellezza, I. Giambanco, A. Minelli, and R. Donato, "Nrf2Keap1 signaling in oxidative and reductive stress," Biochimica et Biophysica Acta (BBA)-Molecular Cell Research, vol. 1865, no. 5, pp. 721-733, 2018.

[15] A. Loboda, M. Damulewicz, E. Pyza, A. Jozkowicz, and J. Dulak, "Role of Nrf2/HO-1 system in development, oxidative stress response and diseases: an evolutionarily conserved mechanism," Cellular and Molecular Life Sciences, vol. 73, no. 17, pp. 3221-3247, 2016.

[16] P. Shaw and A. Chattopadhyay, "Nrf2-ARE signaling in cellular protection: mechanism of action and the regulatory mechanisms," Journal of Cellular Physiology, vol. 235, no. 4, pp. 3119-3130, 2020.

[17] L. I. Xiao, X. Xu, F. Zhang et al., "The mitochondria-targeted anti-oxidant MitoQ ameliorated tubular injury mediated by mitophagy in diabetic kidney disease via Nrf2/PINK1," Redox Biology, vol. 11, pp. 297-311, 2017.

[18] F. Zhao, C. Liu, L. Fang et al., "Walnut-derived peptide activates PINK1 via the NRF2/KEAP1/HO-1 pathway, promotes 
mitophagy, and alleviates learning and memory impairments in a mice model," Journal of Agricultural and Food Chemistry, vol. 69, no. 9, pp. 2758-2772, 2021.

[19] A. Anwar-Mohamed and A. O. S. el-Kadi, "Induction of cytochrome P450 1al by the food flavoring agent, maltol," Toxicology In Vitro, vol. 21, no. 4, pp. 685-690, 2007.

[20] N. Guo, C. Li, Q. Liu et al., "Maltol, a food flavor enhancer, attenuates diabetic peripheral neuropathy in streptozotocininduced diabetic rats," Food \& Function, vol. 9, no. 12, pp. 6287-6297, 2018.

[21] J. Y. Sha, Y. D. Zhou, J. Y. Yang et al., "Maltol (3-hydroxy-2methyl-4-pyrone) slows d-galactose-induced brain aging process by damping the $\mathrm{Nrf} 2 / \mathrm{HO}-1$-mediated oxidative stress in mice," Journal of Agricultural and Food Chemistry, vol. 67, no. 37, pp. 10342-10351, 2019.

[22] Y. Li, J. Xiang, J. Zhang, J. Lin, Y. Wu, and X. Wang, "Inhibition of Brd4 by JQ1 promotes functional recovery from spinal cord injury by activating autophagy," Frontiers in Cellular Neuroscience, vol. 14, article 555591, 2020.

[23] Z. Zheng, Y. Zhou, L. Ye et al., "Histone deacetylase 6 inhibition restores autophagic flux to promote functional recovery after spinal cord injury," Experimental Neurology, vol. 324, article 113138, 2020.

[24] M. Hoop, X. Z. Chen, A. Ferrari et al., "Ultrasound-mediated piezoelectric differentiation of neuron-like PC12 cells on PVDF membranes," Scientific Reports, vol. 7, no. 1, p. 4028, 2017.

[25] A. Blesch and M. H. Tuszynski, "Spinal cord injury: plasticity, regeneration and the challenge of translational drug development," Trends in Neurosciences, vol. 32, no. 1, pp. 41-47, 2009.

[26] M. V. Sofroniew, "Dissecting spinal cord regeneration," Nature, vol. 557, no. 7705, pp. 343-350, 2018.

[27] C. S. Ahuja, S. Nori, L. Tetreault et al., "Traumatic spinal cord injury-repair and regeneration," Neurosurgery, vol. 80, no. 3S, pp. S9-S22, 2017.

[28] B. Fan, Z. Wei, X. Yao et al., "Microenvironment imbalance of spinal cord injury," Cell Transplantation, vol. 27, no. 6, pp. 853-866, 2018.

[29] X. J. Mi, J. G. Hou, Z. Wang et al., "The protective effects of maltol on cisplatin-induced nephrotoxicity through the AMPK-mediated PI3K/Akt and p53 signaling pathways," Scientific Reports, vol. 8, no. 1, article 15922, 2018.

[30] K. G. Lee and T. Shibamoto, "Anti-oxidant properties of aroma compounds isolated from soybeans and mung beans," Journal of Agricultural and Food Chemistry, vol. 48, no. 9, pp. 4290-4293, 2000.

[31] W. Liu, Z. Wang, J. G. Hou et al., "The liver protection effects of maltol, a flavoring agent, on carbon tetrachloride-induced acute liver injury in mice via inhibiting apoptosis and inflammatory response," Molecules, vol. 23, no. 9, p. 2120, 2018.

[32] S. Torres, H. Salgado-Ceballos, J. L. Torres et al., "Early metabolic reactivation versus anti-oxidant therapy after a traumatic spinal cord injury in adult rats," Neuropathology, vol. 30, no. 1, pp. 36-43, 2010.

[33] C. Rios, I. Santander, M. Méndez-Armenta et al., "Metallothionein-I + II reduces oxidative damage and apoptosis after traumatic spinal cord injury in rats," Oxidative Medicine and Cellular Longevity, vol. 2018, Article ID 3265918, 2018.

[34] C. Tonelli, I. I. C. Chio, and D. A. Tuveson, "Transcriptional regulation by Nrf2," Antioxidants \& Redox Signaling, vol. 29, no. 17, pp. 1727-1745, 2018.
[35] T. Suzuki and M. Yamamoto, "Molecular basis of the Keap1Nrf2 system," Free Radical Biology \& Medicine, vol. 88, no. Part B, pp. 93-100, 2015.

[36] D. C. Zhu, Y. H. Wang, J. H. Lin, Z. M. Miao, J. J. Xu, and Y. S. $\mathrm{Wu}$, "Maltol inhibits the progression of osteoarthritisviathe nuclear factor-erythroid 2-related factor-2/heme oxygenase-1 signal pathwayin vitroandin vivo," Food \& Function, vol. 12, no. 3, pp. 1327-1337, 2021.

[37] G. Ashrafi and T. L. Schwarz, "The pathways of mitophagy for quality control and clearance of mitochondria," Cell Death and Differentiation, vol. 20, no. 1, pp. 31-42, 2013.

[38] A. M. Pickrell and R. J. Youle, "The roles of PINK1, parkin, and mitochondrial fidelity in Parkinson's disease," Neuron, vol. 85, no. 2, pp. 257-273, 2015.

[39] P. M. J. Quinn, P. I. Moreira, A. F. Ambrósio, and C. H. Alves, "PINK1/PARKIN signalling in neurodegeneration and neuroinflammation," Acta Neuropathologica Communications, vol. 8, no. 1, p. 189, 2020.

[40] E. M. Fivenson, S. Lautrup, N. Sun et al., "Mitophagy in neurodegeneration and aging," Neurochemistry International, vol. 109, pp. 202-209, 2017.

[41] J. Schultheis, D. Beckmann, D. Mulac, L. Müller, M. Esselen, and M. Düfer, "Nrf2 activation protects mouse beta cells from glucolipotoxicity by restoring mitochondrial function and physiological redox balance," Oxidative Medicine and Cellular Longevity, vol. 2019, Article ID 7518510, 2019.

[42] T. C. Kang, "Nuclear factor-erythroid 2-related factor 2 (Nrf2) and mitochondrial dynamics/mitophagy in neurological diseases," Antioxidants, vol. 9, no. 7, p. 617, 2020.

[43] H. C. Bertrand, M. Schaap, L. Baird et al., "Design, synthesis, and evaluation of triazole derivatives that induce Nrf2 dependent gene products and inhibit the Keap1-Nrf2 proteinprotein interaction," Journal of Medicinal Chemistry, vol. 58, no. 18, pp. 7186-7194, 2015. 